\title{
Molecular biology and the Internet
}

\author{
J Rashbass
}

\section{Introduction}

There are few subjects that have produced such a sustained response in the press over the past few years as the Internet and the Information Super Highway. Every week we are bombarded with details of new facilities that can be found on this vast computer network. In the United States an initiative led by VicePresident Al Gore three or four years ago established the infrastructure necessary for super-computer communications countrywide. As a result, the American public and the medical profession are fully aware of the strengths of this new technology. In Britain, however, we have been slow to exploit the potential of this worldwide communications network. Even though the Internet has now entered the political debate, lack of a suitable national computer network and a centrally co-ordinated policy has meant that we are rapidly falling behind in the range of resources available to us at our desks. Despite the amount written about the Web in the medical literature in Britain, the number of medical users remains small. In these few columns I should like to provide a glimpse of the variety of resources that are available to the molecular and diagnostic histopathologist, the limitations of the Internet, and what this invaluable facility promises in the future.

\section{What is the Internet?}

The Internet is a worldwide collection of computers that share a common and fairly simple communication protocol called TCP/IP (Transmission Control Protocol/Internet Protocol). There is no restriction on the types of wires or links between these computers and they range from the radio waves that link satellites to the simple telephone wires that connect our homes. Each machine on the Internet, and now there are about 31 million connected users, has its own unique identity called an IP (Internet Protocol) number. Information leaving that machine, or going to it, is identified electronically by the presence of this number. Once assigned an IP number, which might be from a commercial vendor (an Internet provider) or through the computer services administered by larger institutions, the user has access to all the facilities on the Internet. This may simply be electronic mail, where messages and documents can be sent from one computer user to another, or bulletin boards where users with a common interest can post and read articles. Users also have access to the vast array of more sophisticated facilities on the World Wide Web.

The World Wide Web has come to dominate the use of the Internet. It is its simplicity and the fact that it incorporates text, images, video, and sound that makes it so attractive. Fundamental to the Web is the concept of "hyper- text". This allows the text within one document to be linked with another document, picture or video which can be on any computer anywhere on the Web. The key to this is a hidden link or Uniform Resource Locator (URL) buried within the text. This means of "publishing documents" involves converting them into a modified format called HyperText Markup Language (HTML); there are many tools now available to allow a word processor document to be converted into this format within a matter of seconds. Information on the Web is provided from computers called Web Servers or Web Sites and accessed by a program called a browser, run on the user's local computer. The majority of resources I will discuss are on the Web and the URLs are given in the appendix.

\section{Molecular biology databases}

The Internet has been an essential tool for molecular biologists for many years. It provides the communication network to search for DNA and protein sequence data at sites such as the Sanger Centre in Britain ${ }^{(a)}$, GenBank ${ }^{(b)}$ in the USA or EMBL ${ }^{(c)}$ in Heidelberg. Sequences can be compared with those already known in the database and homologies rapidly identified (fig 1). At present, the GenBank database contains nearly 500000 DNA sequences, totalling more than 350 million nucleotide bases from over 11000 biological species. New human DNA sequences are being added to the database at a rate of 1500 a day. ${ }^{1}$ At this rate of increase, sending the information by post on digital tapes or CD-ROMs is clearly an inefficient means of distribution. Using the Web gives access to the information from any site in the world within minutes. Programs such as Entrez ${ }^{(d)}$ allow the user to search for sequences in GenBank and, at the same time, find the Medline records from the National Library of Medicine with references and abstracts that refer to that particular sequence. Entrez will also allow the user to visualise the three dimensional structure of the proteins that interest $\mathrm{him} / \mathrm{her}$. Searches for sequence similarity can be made with programs such as BLAST $^{(e)}$ (Basic Local Alignment Search Tool), in which the test sequence is sent to a computer at the National Centre for Biotechnology Information in Bethesda (Maryland, USA). ${ }^{2}$ The alignment is performed within a matter of minutes and the answer is e-mailed back to your computer.

Features such as these highlight the power of the Internet. The ability to perform complex computational calculations is no longer limited by power of the user's computer. The request and the necessary data are transferred to a centrally maintained computer anywhere on the Web, where the power is adequate to perform
Cambridge CB2 2QQ email:

jem@mole.bio.cam.ac.uk 


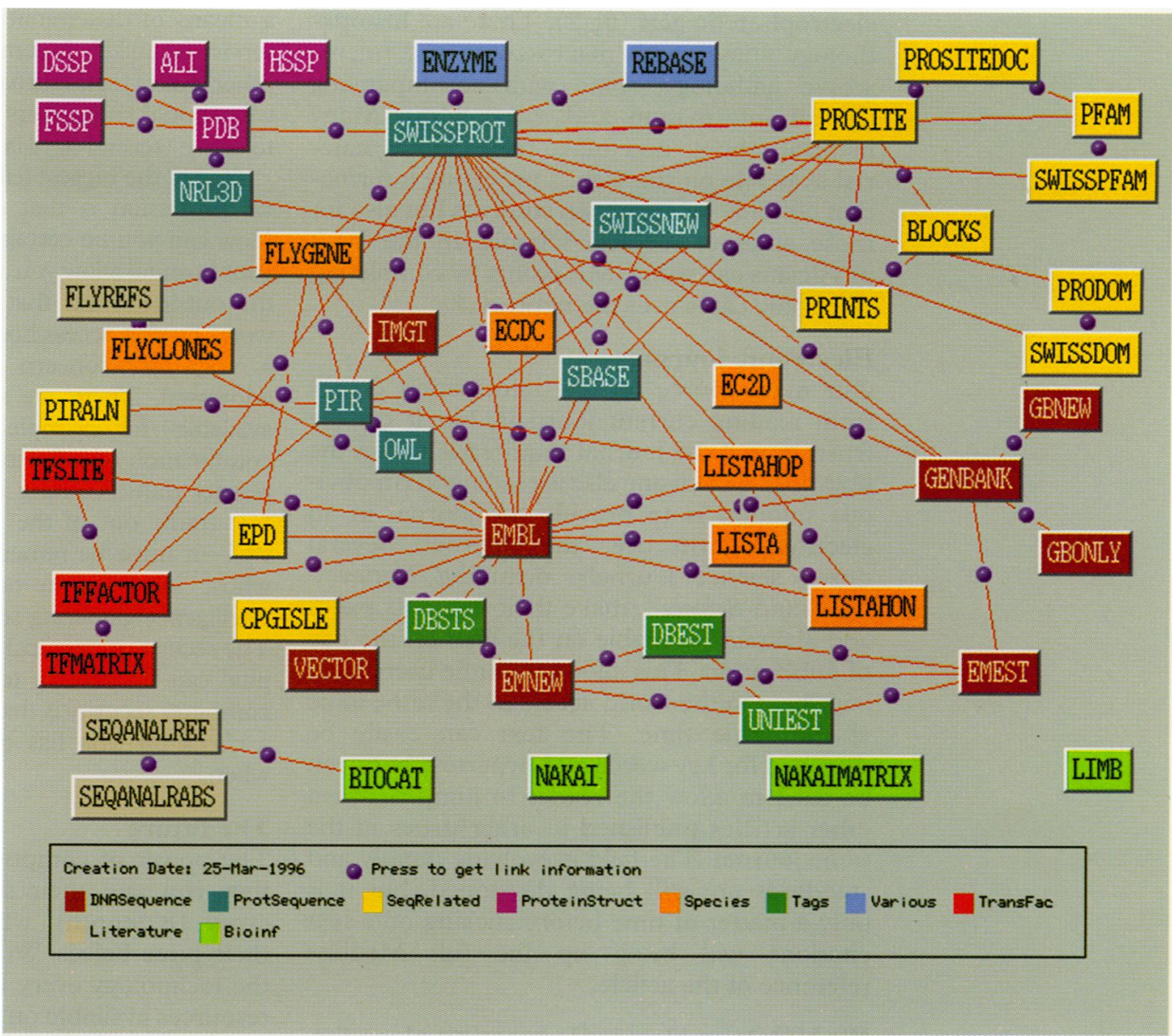

Figure 1 A schematic diagram of the molecular biology databases available on the Web from the Sanger Centre in the UK (http://www.sanger.ac.uk/srs/srsc). This image is an "active map"-clicking on an icon in the picture takes the user straight to that database, where electronic forms then allow him/her to enter questions into the computer to perform searches and retrieve data. Reproduced with kind permission of Thure Etzold.

the tasks. The answer, be it a picture, text or a video, is simply e-mailed back to your computer.

\section{Biomedical databases}

The Internet is the largest "single" collection of documents ever accumulated. All the information is accessible from the user's computer and constitutes a vast electronic library which is relatively easily searched, rapidly updated and

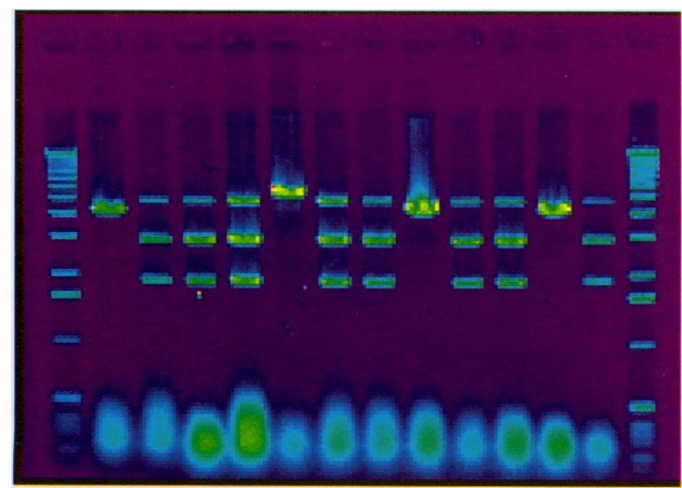

Figure 2 There are several Web sites which provide electronic access to laboratory protocols for molecular biology. This one is run by Mark Strom at the Microbial Pathogenesis/Utilization Research Division, Northwest Fisheries Science Center (NMFS/NOAA) in the USA (http://research.nwfsc.noaa.gov/protocols.html). There is a range of methods from full techniques to helpful tips, all instantly available on the user's Web browser. An electronic form allows users to submit additional comments or techniques. Reproduced with kind permission of Mark Strom. allows a single resource to be accessed by many people at the same time. One of the most elegant medical facilities available, which has evolved with the development of the Internet and the World Wide Web, is Victor McKusick's online version of Mendelian Inheritance in Man $(\mathrm{OMIM})^{(\mathrm{f})}$. Mendelian Inheritance in Man was first published as a two volume work in the early 1960s and has been constantly updated since then. The first electronic version appeared in 1985 and could be searched using tools such as the Internet Gopher, a simple but very powerful text retrieval tool. With the introduction of the World Wide Web and the graphical browsers which support pictures and sound, OMIM has been updated to include video and audio clips. It is now possible to hear the cry of a baby with "Cri du chat" syndrome or see the choreoform movements of a patient with Huntington's disease. The OMIM database gives extensive information on the classification of genetic diseases, clinical presentations and genomic mapping of the disease locus. The database is updated continuously and provides probably the best 'first port of call' when looking for information on hereditary conditions. ${ }^{3}$

There are many other biomedical resources on the Web and many have a molecular or pathological bias. There are on-line handbooks $^{(\mathrm{g})}$ of laboratory protocols with clear instructions on areas from cloning to running 
electrophoretic gels (fig 2). There are histopathology atlases with macroscopic and microscopic pathology, some with accompanying clinical information and $x$ ray films. Many medical schools now make their course material available on the $\mathrm{Web},{ }^{\text {(h) }}$ providing information not only for their own students but also for those at other medical schools. In the USA physicians can gain CME points by completing electronic cases over the network. ${ }^{(i)}$

\section{Electronic journals}

Our main source of new information comes from reading current journals, but it can be frustrating if the journal required is not in the library or if someone else is reading it. An obvious solution is to provide the journal in an electronic form over the Internet. Several dozen science journals, including Nature ${ }^{(j)}{ }^{4}$ $\mathrm{Cell}^{(\mathrm{k})}$ and Science ${ }^{(\mathrm{l})}$, make their contents pages and abstracts available on the Web on the day of publication. This allows multiple users from anywhere in the world access to the same issue at the same time. The text can easily be searched for key words and hypertext links can be used to allow the reader to jump between other articles published in other issues of the same journal. The field is evolving rapidly and standards are still being developed, but it is only a matter of time before clicking on a text citations will bring up the full Medline reference of the article.

\section{BioMOO: an electronic meeting place for biologists}

The majority of Web resources provide information for individual users. There is little scope for immediate interaction with other Web users. One exception is a MOO (Multiuser Dimension Object Orientated), a virtual reality meeting place at which multiple users can be present at once. $\mathrm{BioMOO}^{(\mathrm{m})}$ is one such facility for biological scientists, in which users from around the world can log onto a computer at the Weizmann Institute in Israel and converse with each other, hold symposia and read notice boards, posters and job advertisments. $^{5}$ The structure of the MOO illustrates the beauty of the Web because the images and slide collections found in BioMOO are stored on a separate computer at CalTech in California and the two sites are linked seamlessly by hypertext.

\section{Too much of a good thing?}

Initial enthusiasm about the Web must be tempered by reality. There are times when the network is so slow that it is unusable and while the number of resources is remarkable, there is in general no peer review system for determining the quality or the reliability of the information. There are reputable institutions which make information available, but there is also much more information produced from dubious sources on the 'fringe'. Sorting between this information can be difficult, time consuming and costly, especially if you pay by the minute for your connection. At present, most of the resources on the Web are free and without copyright, provided by non-commercial organisations and individuals. Many who use the Web are funded by research grants and use the Internet as a means of disseminating their knowledge. The service is unlikely to remain free. After all, if a publisher makes their journal available free on the Web why should subscribers pay for the paper version to arrive (several days later) on their desk?

One of the biggest fears expressed by the medical profession is that of patient confidentiality. How can you be certain that if the computers in your hospital allow you access to information from the outside world that others from the outside world cannot access hospital information? This is a very real concern and one that is being addressed. There are several security measures available - for example, a 'firewall', in which a combination of software and hardware is used to restrict traffic coming into a site while allowing free traffic out of the site. These systems work well-it must be remembered that organisations whose job it is to be paranoid, such as the intelligence gathering services, make use of them. The security of medical records even within a hospital can be difficult to achieve; one strength of computer security is that you can at least maintain a check on who has been looking at what and when.

\section{The future}

The power of computers, the speed at which they run and their storage capacity doubles every 18 months, while in the same period their price halves. With this rapid advance in the technology every few weeks, the variety of resources available on the Internet is changing. Encryption technology is being developed which, in the next few months, will allow the secure use of credit card numbers on the Web. Suddenly, a whole world of commerce will appear on the Internet. The same technology could permit medical information to be sent securely over the Web and improvements in communication technology in the next few years will allow real-time video information to be transmitted. We will all be attending virtual conferences and seminars within the next five years.

\section{Conclusions and suggestions}

In Britain we are being bombarded by information about the resources available on the Web; however, the infrastructure necessary is unavailable to many in the medical profession. Facilities which would be of considerable benefit to all pathologists are restricted to a few isolated enthusiasts. Those of us who are fortunate enough to be able to use the Internet easily, support the idea of an official body to co-ordinate and represent the interests of all pathologists. Communication and information are fundamental to all branches of medical practice and it is clear that pathologists throughout Britain should not be denied access to these facilities.

1 Boguski MS. Molecular Medicine - Hunting for genes in computer databases. $N$ Engl f Med 1995;333:645-6.

2 Brenner SE. BLAST, Blitz, BLOCKS and BEAUTY: sequence comparison on the Net. Tends Genet 1995; 11:295-6.

3 Rashbass J. Online Mendelian Inheritance in Man. Trends Genet 1995;11:291-2.

4 Maddox J. Nature on the Internet (at last)! Nature 1995;377:475.

5 Rashbass J. The Web goes MOO. Molecular Medicine Today 1995;1:114-17. 


\section{Appendix}

$\begin{array}{ll}\text { (a) } & \text { Sanger Centre } \\ \text { (b) } & \text { GenBank } \\ \text { (c) } & \text { EMBL } \\ \text { (d) } & \text { Entrez } \\ \text { (e) } & \text { BLAST } \\ \text { (f) } & \text { OMIM } \\ \text { (g) } & \text { Protocols } \\ \text { (h) } & \text { Teaching } \\ \text { (i) } & \text { CME units } \\ \text { (j) } & \text { Nature } \\ \text { (k) } & \text { Cell } \\ \text { (l) } & \text { Science } \\ \text { (m) } & \text { BioMOO }\end{array}$

http://www.sanger.ac.uk/searching.html http://www.ncbi.nlm.nih.gov

http://www.embl-heidelberg.de/srs/srsc

http://atlas.nlm.nih.gov:5700/Entrez/index.htm

http://www.ncbi.nlm.nih.gov/BLAST/

http://www3.ncbi.nlm.nih.gov/Omim/

http://research.nwfsc.noaa.gov/protocols.html

http://www.primenet.com/ gwa/med.ed/courses.html

http://ns.gamewood.net/pathcase/nephpath.htm

http://www.nature.com

http://www.cell.com

http://www.aaas.org/science/science.html

http://bioinfo.weizmann.ac.il:8888 